\title{
An integrated approach for the design of emulsified products
}

\author{
Kontogeorgis, Georgios M.; Mattei, Michele; Ng, Ka M.; Gani, Rafiqul
}

Published in:

A I Ch E Journal

Link to article, DOI:

10.1002/aic. 16363

Publication date:

2019

Document Version

Peer reviewed version

Link back to DTU Orbit

Citation (APA):

Kontogeorgis, G. M., Mattei, M., Ng, K. M., \& Gani, R. (2019). An integrated approach for the design of emulsified products. A I Ch E Journal, 65(1), 75-86. https://doi.org/10.1002/aic.16363

\section{General rights}

Copyright and moral rights for the publications made accessible in the public portal are retained by the authors and/or other copyright owners and it is a condition of accessing publications that users recognise and abide by the legal requirements associated with these rights.

- Users may download and print one copy of any publication from the public portal for the purpose of private study or research.

- You may not further distribute the material or use it for any profit-making activity or commercial gain

- You may freely distribute the URL identifying the publication in the public portal

If you believe that this document breaches copyright please contact us providing details, and we will remove access to the work immediately and investigate your claim 
based design stage, structured databases, dedicated algorithms and a property model library are employed for designing a candidate base case formulation. Finally, in the experiment-based verification stage, the properties and performances of the proposed formulation are measured by means of tailor-made experiments. The formulation is then validated or, if necessary, refined thanks to a systematic list of actions. All these tools have been implemented as a new template in our in-house software called the Virtual Product-Process Design Laboratory and have been illustrated via a case study (a hand-wash detergent) where the complete methodology (all three stages) is for the first time applied.

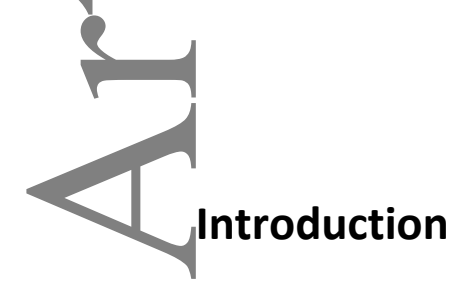 Process Systems Engineering (PSE), now a well-established field, has had a remarkable impact on the chemical and related industries ${ }^{1}$, introducing methodologies, which are now employed Throughout the industry world-wide. Since the 1990's, however, interest in chemical product design and engineering has emerged stimulated by contributions to areas such as selection and design of solvent systems and working fluids.

Background in transport phenomena and thermodynamics has been applied to understand the Processes characterizing the properties and performances of chemical products. The process systems engineering community contributed to this development by introducing mathematical modeling, especially in the applications where the product properties and performances can be related to the molecular structure of chemicals. Moreover, the process systems engineering community satisfied the necessity of capturing the knowledge of product designers, storing it and then providing it in terms of explicit knowledge to be manipulated to perform predictions that can be used for the design and development of innovative products ${ }^{2}$.

This article is protected by copyright. All rights reserved. 
The complexity of chemical product design derives from the recent shift that is observed in the chemical industry: from materials valued for their purity, such as, the commodities, to materials sold for their performance behavior, such as consumer products ${ }^{3}$. There are, in fact, limited raw materials, which are processed to obtain the commodity products (basic products). Specialty chemicals (intermediate products) are then manufactured from the commodities, and finally a very large portfolio of higher value products (refined chemicals and consumer products), are obtained by processing and/or combining the chemicals of the previous classes. The number of products belonging to each category grows exponentially from around 10 for the raw materials, to almost 30,000 in the last class of higher value added products ${ }^{4}$.

Usually, the last types of chemical products are classified into three categories ${ }^{5}$ :

- Devices: miniature processes which accomplish a particular chemical transformation at a small scale, as an artificial kidney, or a mosquito repellent vaporizer;

- Molecules: most often exemplified by pharmaceuticals, where the key is not process cost or convenience but discovery of the active compound in the first place;

- Structures: includes various consumer products, such as cosmetic and food products, where the key is the product function.

The class of structures includes the wide sub-class of the formulated products. These consist of Pharmaceuticals, paints, creams, detergents, pesticides, etc., in which 5 to more than 20 ingredients are usually present, representing a wide range of chemical compounds, such as, polymers, surfactants, solid particles, solvents, pigments, and aromas.

Irrespectively to the product type, the common practice, in the development of such products, is still the experiment-based and trial-and-error approach, as explained in well-known textbooks in the field ${ }^{5,6}$. However, a systematic integrated procedure, where candidate higher added value 
products are designed through a model-based methodology, and then validated and/or refined by means of dedicated experiments, represents an efficient alternative, with respect to time and resources, speeding up the product development process.

(1) An even more complete integrated approach for such complex products would include an integration of the product design and manufacturing (production) aspects, including a complete

simulation analysis of the latter. This is, however, beyond the purpose of this work, but it will be discussed further in the manuscript.

1

2

Among the consumer chemical-based products, many are of the formulation type: homogeneous liquid formulation where solid active ingredients are kept in solution by a proper solvent mixture; suspensions containing insoluble chemicals dispersed in the liquid mixture with the help of proper dispersing agents; emulsions where immiscible liquids are kept together through selected emulsifying agents; solid products such as pharmaceutical tablets or soap bars. The performances 1 of such products are related not only to the presence of active ingredients and additives in the formulation, but also to the product's structural and material properties ${ }^{7}$.

Among this category of products, emulsified formulated products, which are the focus of this work, are very relevant, with wide application in the food and cosmetic industries. Emulsions are C defined as mixtures of two normally immiscible liquids, kinetically stabilized by emulsifying agents (most often surface active agents, that is, surfactants) that lie at the interface between the two liquid phases. Active ingredients and additives are then dissolved in the continuous and/or in the dispersed phases, according to the needs of the product.

With regards to emulsified formulated products, methodologies have been recently proposed to simultaneously tackle product and process design, with particular attention to cosmetic 
emulsions $^{8}$, and also to integrate multi-disciplinary optimization, by adding the complexity of price-competitive markets ${ }^{9}$.

However, there is a lack of studies where process systems engineering tools are systematically applied for the solution of problems involving the design of emulsified formulated products. In this manuscript, we present a systematic methodology for the design of emulsified formulated products.

- Prior to presenting this methodology in detail, the general outline is first given.

1

As mentioned above, chemical products as mixtures and blends are usually considered when a single molecule is unable to provide all the desired product functions, or perform all the desired

process tasks. Solvent mixtures, tailor-made fuels, lubricants are examples of blended chemical products.

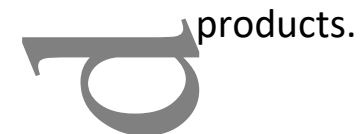

(1) A wide variety of computer-aided methods and tools have been developed for design of chemical products represented by the properties of a single molecule. These methods are classified under CAMD (Computer Aided Molecular Design) and they follow the same main steps: generate feasible chemical structures, estimate the thermo-physical properties through property models, and select (2) the molecules that match the desired targets. The main features for these methods and tools are that building-blocks based methods are used to represent the molecular structures and their contributions are used to estimate the $\operatorname{target}^{10}$.

For the computer-aided mixture-blend design $\left(C A M^{b} D\right)$, the properties and algorithms are different, even though similar building-block based property models may be used. Here, the properties depend on the mixture composition, and the design algorithm needs to identify the molecules and their compositions in solution matching the target properties. Systematic decomposition based solution approaches are usually employed to manage the complexity of 
these design problems efficiently, by reducing the search space ${ }^{9}$. Recently, direct solution approaches of the MINLP (mixed integer non-linear programming) problem formulation have been reported $^{19,20}$.

Three types of solution approaches to product design are usually identified ${ }^{11}$ :

- Experiment-base (often partially based trial-and-error) approach: this approach is employed when mathematical models for the estimation of target properties are not available. A large number of consumer products are currently developed through this approach, where past knowledge and experience are crucial;

- Model-based approach: when validated mathematical models for the estimation of the target properties are available, a list of feasible candidates is efficiently and quickly generated and tested. CAMD and $C A M^{b} D$ enter in this type of solution approach to product design;

- Integrated experiment-modelling approach: this approach is used when mathematical models are not available for all the target properties. The design problem is decomposed into a hierarchical sequence of sub-problems: as one goes from the outer levels to the inner levels, the number of candidate decreases and the inner levels employ experiments for the final product refinement and/or validation.

In general, the first approach is particularly reliable but extremely time expensive and innovative solutions are rarely found. The second approach, on the other hand, is characterized by very limited development time and consumption of resources, but it is highly uncertain and usually it needs external validation.

The third approach, instead, represents an ideal combination of the two: high chances for innovation are guaranteed by the model-based structure, and the development time and the 
consumption of resources are kept low, as the experiments are carefully designed for final refinement.

The objective of this manuscript is exactly to present a systematic methodology for emulsified product design using this third approach as well as present a complete case study where this approach is used.

The proposed methodology is hereafter presented in the next section, followed by the case study results. Several detailed aspects are presented in the Supplementary Material.

\section{1 \\ 2

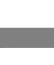 \\ Methodology and Tools}

The overall framework and integrated methodology proposed in this work for the design of emulsified formulations is illustrated in Figure 1. The framework, which includes model-based and experiment-based stages interacting with each other, is inspired by the approach proposed by $\mathrm{Ng}$, 1

Gani and Dam-Johansen ${ }^{11}$ and its extension for the solution of problems involving homogeneous Tormulated products by Conte et al. ${ }^{12}$.

All three stages play very important roles and crucial in this methodology are also the databases and property models used especially in the model-based design stage. While aspects of this methodology and some case study applications (limited to stages 1 and 2) have been briefly presented previously ${ }^{13-16}$, a more comprehensive presentation is offered in this study as well as a fully worked out case study, which will include all three stages of the product design outlined in Figure 1. First of all, in this section, a presentation of all three stages is given, including their interrelations.

This article is protected by copyright. All rights reserved. 


\section{Framework and Methodology}

The systematic methodology, integrating model-based as well as experiment-based techniques, requires the definition of the product of interest as the input, and then gives as the output a validated emulsified formulation, containing a list of ingredients together with their relative concentrations. There is a close interaction between all stages, especially stages 2 and 3 , as shown in Figure 2. The necessary methods and tools may differ for each of the stages.

The problem definition stage is very important, as it is first in the hierarchical structure of the ()

methodology. In some cases backwards interactions from the other stages may be difficult or time consuming, and any decision taken at this level influences the decisions taken in the following steps. However, in cases of generation of too few or too many candidates, the problem definition should be refined. At this point, a list of target thermo-physical properties (including target values and and boundaries of acceptance) and a list of necessary categories of ingredient are generated, given the type of product of interest as the input. The main tool used in this stage is the knowledge base.

model-based stage takes the results of the problem definition stage and, through the use of property models, structured databases and dedicated algorithms, converts them into a candidate emulsified formulated product to be verified in the final stage, that is, a list of candidate ingredients and relative concentrations are determined. In order to reliably screen thousands of candidates while applying the "reverse design" technique, the model-based stage employs a decomposition strategy, so that the solution method is divided into a set of sub-problems to be solved individually. A wide range of tools takes part in this stage: property models, structured databases and algorithms.

This article is protected by copyright. All rights reserved. 
Finally, the experiment-based stage is in charge of verifying the formulated product proposed by the previous stage, through focused experiments. A list of actions is also generated so that, if the results of these experiments do not match with the expected results provided by the previous stage, appropriate corrections can be taken.

As illustrated in Figure 2, the interaction between the model-based stage and the experimentbased stage is not unilateral, and iterations within the two stages are required if the formulation proposed by the model-based stage is not validated in the experiment-based stage. Iterations are necessary until a candidate formulated product generated by the model-based stage is verified by the experiment-based stage.

The main objective of the overall integrated methodology is to quickly and efficiently screen between many candidates by means of mathematical models and algorithms, so that the valuable experimental resources are reserved for the final verification and possible refinement. This, however, is possible only if a set of product needs is generated. Such needs include both necessary categories of ingredients to be included in the candidate emulsified formulated product, and tariget properties to be satisfied by the above-mentioned components.
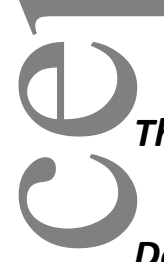

\section{The Problem Definition (Knowledge base) Stage 1}

\section{Description of Stage 1}

The first stage of the integrated methodology for the design of emulsified formulated products consists of problem definition and it is illustrated graphically in Figure 3. Based on the knowledge base (databases and property tools, see "Knowledge-based of chemical product design" in Supplementary Material), this stage defines a set of target properties and ingredients relative to the product to be designed. It involves three main tasks, containing different sub-tasks, as shown in Figure 3.

This article is protected by copyright. All rights reserved. 
In this first task, the consumer needs $\left(\psi_{\mathrm{i}}\right)$ relative to the product to be designed are collected from the knowledge base and they are divided into main $\left(\psi_{M}\right)$ and secondary $\left(\psi_{S}\right)$ needs. The consumer needs are defined as those attributes that consumers need and/or want from a product in order to buy it. The consumer needs include a wide variety of product attributes, ranging from cosmetic properties and sensorial factors, to safety and toxicity related property, to product form and phase stability. Data and information relative to the collection of the consumer needs for a range of formulated products can be retrieved mostly from customer surveys but also from literature, patents, real products, insight, industrial experience and common sense.

The main needs $\left(\psi_{\mathrm{m}}\right)$ are defined as the main reasons for which consumers would buy the product, while the secondary needs $\left(\psi_{\mathrm{s}}\right)$ are defined as accessory product attributes, which can drive consumers to prefer a product, as compared to another, but the absence of which does not influence the minimum product performances. For example, the main need of an insect repellent

(1) product is to repel mosquitos, while the main need of a UV sunscreen is to provide protection from the UV radiations. The secondary needs are usually related to the form of the formulated product (liquid, solid, emulsion, spray...), safety, toxicity, cosmetic properties (odor, color...), and so on. For example, between the secondary needs of an insect repellent product, one can identify a pleasant odor and the ease of spreading or spraying, while in relation to a UV sunscreen, a pleasant skin feeling and the prevention of skin ageing are among the secondary needs.

In the second task, first those consumer needs which cannot be translated into a set of thermophysical properties are identified e.g. color, odor or even ability to repel mosquitos for insect repellent lotions. These needs are translated into properties that directly link to categories of ingredients $\left(\xi_{i}\right)$ that are known to be able to satisfy these needs. Then, the distinction between main and secondary consumer needs is used to distinguish between the active ingredients $\left(\xi_{\text {AII }}\right)$ and the additives $\left(\xi_{\mathrm{ADD}}\right)$. The active ingredients $\left(\xi_{\mathrm{Al}}\right)$ are defined as those ingredients that are able 
to satisfy the properties connected to the main product needs $\left(\psi_{\mathrm{m}}\right)$, while the additives $\left(\xi_{\mathrm{ADD}}\right)$ are defined as those ingredients that are able to satisfy the target properties connected to the secondary product needs $\left(\psi_{\mathrm{s}}\right)$.

Indeed, despite the difficulties with these needs, there are categories of ingredients that may be able to satisfy them e.g. coloring agents (dyes or pigments), in relation to the main need of a paint. There are cases, however, where more classes of ingredients correspond to a single consumer need, such as in relation to the protection from UV radiations. In fact, there are very few chemicals which have been proven to be effective in screening the skin from both UV-A and UV-B radiations, therefore UV-A as well as UV-B absorbers and/or filters are necessary.

In the design methodology, then, these consumer needs are translated into target properties, even though no quantitative models are available. These properties are defined in this work as target properties I, in order to distinguish them from those properties (target properties II) for which models are available. For the application of such a sub-task, a reliable knowledge base is necessary, discussed in the next section.

(2)

of thermo-physical properties $\left(\zeta_{i}\right)$, that is, target properties are identified. Then, they are divided between those relative to the active ingredients $\left(\zeta_{\mathrm{Al}}\right)$, those relative to the additives $\left(\zeta_{\mathrm{ADD}}\right)$, those relative to the final product $\left(\zeta_{\mathrm{PROD}}\right)$, and those relative to all the ingredients $\left(\zeta_{\mathrm{ALL}}\right)$. Finally, target values and boundaries of acceptance are set for each target property. Examples of target properties are the surface tension and the critical micelle concentration in relation to the foamability of a hand-wash $\left(\zeta_{\mathrm{Al}}\right)$; the solubility in the solvent mixture, quantified by means of the solubility parameters $\left(\zeta_{\mathrm{ADD}}\right)$, the flash point and the toxicity parameter, that are fundamental properties, in relation to safety and toxicity issues $\left(\zeta_{\mathrm{ALL}}\right)$ and finally bulk mixture properties such as 
density, viscosity and surface tension, derived from consumer needs like spread-ability, sprayability are related to the overall product ( $\left.\zeta_{\mathrm{PROD}}\right)$.

For the set of target properties $\zeta_{\mathrm{i}}$ the target value $\left(\zeta_{\mathrm{i}, \mathrm{T}}\right)$ and the boundaries of acceptance $\left(\zeta_{\mathrm{i}, \mathrm{L}}, \zeta_{\mathrm{i}, \mathrm{u}}\right)$ are defined. The target values are the numerical values that certain properties are desired to match, while the boundaries of acceptance are the lower and upper numerical values that certain properties are required not to go beyond. The boundaries of acceptance are usually applied for screening purposes, while the target values are commonly used as performance index for ingredient selection. Target values are not always required, while two boundaries of acceptance are necessary for each target property. In case only a lower or an upper boundary can be set, the second is set to a very large positive value, or to a very large negative value, respectively.

\section{Stage 2: Model-based stage including algorithm (Methods and Tools)}

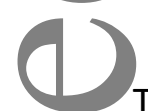

The work-flow of this model-based is shown in figure 4, together with the various sub-tasks. 1

The mixture design problem addressed by this algorithm is a typical reverse design problem where, the design of solvent mixtures satisfying a set of constraints representing the target properties are determined and ranked according to a specific objective function, or performance index.

Task 2.1

The objective of task 2.1 is to select the most advantageous active ingredients $\left(\xi_{\mathrm{Al}}\right)$ and retrieve their properties using databases and property models. For the relevant thermo-physical properties of the candidate Als, if data are not available in the database, they are calculated through dedicated property models.

This article is protected by copyright. All rights reserved. 
Prior to performing Task 2.1, the relative databases to the necessary categories of active ingredients $\left(\xi_{\mathrm{Al}}\right)$ are retrieved from the database library or, if not available, are generated with information from literature, patents, real products and experience. The database needs to be filled with all the relevant properties for the selection task, that is, at least all the target properties relative to the active ingredients $\left(\zeta_{1}\right)$ and to all the ingredients $\left(\zeta_{3}\right)$. If these properties are not available, property models are applied to fill the gap in the property table. The screening process is carried out by screening out all the ingredients of the database whose numerical value of any of

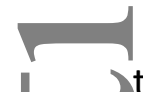
the target properties is lower than the lower boundary of acceptance $\left(\zeta_{i, L}\right)$, or higher than the upper boundary of acceptance $\left(\zeta_{\mathrm{i}, \mathrm{U}}\right)$. Those ingredients for which it has not been possible to retrieve either to calculate all the necessary target properties are excluded from the selection process.

\footnotetext{
From the short-list of candidate active ingredients generated, the most appropriate ingredient to

(1) be added to the formulation is selected, based on a performance index, that is typically one or combinations of the following criteria: effectiveness, safety and health, environment, and/or cost.
}

The target properties usually employed in this sub-task are $\zeta_{1}$ for the effectiveness, the flash-point for the safety, the toxicity parameter for the environment, and the market price for the cost.

()

Finally, all properties for the active ingredients are retrieved either via databases or via property models. It is also important to collect information about the solubility of each active ingredient (usually quantified by means of the Hildebrand and/or Hansen solubility parameters), density, viscosity, and any other thermo-physical properties that can influence the bulk properties $\zeta_{4}$. Finally, as most often models able to relate the active ingredients performances $\left(\zeta_{1}\right)$ to their concentration in the solvent mixture are not available, this information needs to be added to the database so that during problem solution it can be retrieved in terms of recommended concentrations of each of the selected active ingredients. Such concentrations are eventually 
refined during the experiment-based stage, in order to find the optimal solution, which corresponds to the minimum amount of the active ingredients needed and thereby satisfying the desired performances $\left(\zeta_{1}\right)$.

\section{Task 2.2}

Task 2.2 is very crucial, as the optimal solvent mixture in the emulsified form is designed, that is, the most advantageous aqueous solvents, organic solvents, surfactant system and their composition are determined. First, the relative structured databases are selected, then the 1 necessary property models are chosen from the property model library, and finally the emulsified solvent mixture design (EMUD) algorithm is applied, together with the information generated in Stage 1 relative to the overall product $\left(\zeta_{4}\right)$ and to all the ingredients $\left(\zeta_{3}\right)$. Every chemical in the database for which any of the above mentioned target properties is missing, is screened out from the design process.

In a mixture design calculation, pure component property models and mixture property models 1

are simultaneously applied to solve the reverse problem, that is, given a set of constraints $\left(\zeta_{\mathrm{i}, \mathrm{T}}, \zeta_{\mathrm{i}, \mathrm{L}}\right.$, $\left.\zeta_{i, U}\right)$ on a set of target properties $\left(\zeta_{4}, \zeta_{3}\right)$, determine the solvent mixture that match the constraints. For some target properties, different property models can be applied. This is often true, for example, for mixture properties, where a linear mixing rule or rigorous models (non-linear mixing rule) may be applied. It is necessary here to choose which property models are to be applied by the EMUD algorithm for the solution of this reverse problem. It is a common practice, however, to use linear mixing rule models (during candidate generation) since the rigorous models are computationally expensive and it is tedious to apply them to several candidate solvent mixtures. On the contrary, in the verification step, where the thermo-physical properties of the chosen solvent mixture are calculated, rigorous models must be applied. 
The complete EMUD algorithm is shown in Figure 5.

The emulsified solvent mixture design (EMUD) algorithm is applied using all the aforementioned information (databases, relevant target properties with target values and boundaries of acceptance). It has to be underlined that applying the constraints on the target properties relative to the product $\left(\zeta_{4}\right)$ to the solvent mixture design, corresponds to assuming that the bulk properties of the product are identified with those of the solvent mixture. Such an assumption is necessary, in order to reduce the complexity of the problem, but for most of the consumer oriented products, the solvent mixture represents $70-90 \%$ (in mole percentage) of the whole product, therefore this assumption is very close to reality. The output of this sub-task is a candidate emulsified solvent mixture, with its optimal composition, as defined by the EMUD algorithm. Finally, rigorous mixture property models are applied to the candidate emulsified solvent mixture, in order to calculate and collect all its relevant properties. In this sub-task, it is possible to compare (1) the results obtained with the application of the linear mixing rule to a certain set of target J 1 properties with those obtained with the rigorous models. More important, here it is necessary to check that, by applying the rigorous models, the target properties $\left(\zeta_{4}, \zeta_{3}\right)$ are still matching the given constraints $\left(\zeta_{i, T}, \zeta_{i, L}, \zeta_{i, U}\right)$. If this is not true, then the candidate solvent mixture is rejected, and the EMUD algorithm is run again, without the solvent mixture which has just been excluded.

\section{Task 2.3}

In task 2.3 , the most suitable chemicals necessary to satisfy the secondary consumer needs $\left(\psi_{\mathrm{s}}\right)$ are selected. First, the necessary databases relative to the categories of ingredients identified with $\xi_{2}$, are retrieved. Then, the most advantageous chemicals for each category of ingredients are selected by means of an appropriate performance index (same as in task 2.1 ) and finally, all the relevant thermo-physical properties of the candidate additives are retrieved from the databases, or if not available, they are calculated through dedicated property models. 
These databases need to contain all the relevant properties for the selection task, that is: the target properties relative to the additives $\left(\zeta_{2}\right)$, those relative to all the ingredients $\left(\zeta_{3}\right)$, and solubility information, usually quantified by Hansen and Hildebrand solubility parameters. Differently than with active ingredients, in fact, the additives are selected after the solvent mixture, therefore appropriate constraints on the solubility parameter are needed.

Finally, all the thermo-physical properties that are required in the next steps of this model-based stage are retrieved, including properties like density, viscosity, surface tension, etc., as they influence the overall product properties $\zeta_{4}$. Even for additives, there are very few cases for which it s possible to relate the performances of the different ingredients $\zeta_{2}$ with their concentrations in the product. Therefore, it is necessary in this sub-task to define a recommended concentration, that can be eventually further optimized in the experiment-base stage.

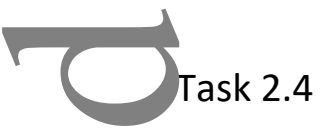

(1)

The fourth task is a model-based stability check on the overall candidate formulation. At the same 1

time, the relevant thermo-physical properties of the overall product are calculated and compared writh the constraints: $\zeta_{\text {PROD }}$. In this task, all the formulation ingredients are included in the mixture property calculations. In fact, the addition to the formulation of certain categories of additives can dramatically change both the overall product properties, and the product stability. If this is the case, another additive may need to be selected.

The additives, as it can be understood, are necessary in the formulation, so that some of the secondary consumer needs are satisfied. There are categories of additives that are responsible for extreme effects on some overall thermo-physical product properties, even if added in very low concentrations. Therefore, it is necessary to check, after all the ingredients of the formulation have been chosen, if the overall product target properties $\left(\zeta_{4}\right)$ are still in agreement with the 
boundaries of acceptance previously set, i.e., if the assumption that the solvent mixture is responsible for the overall product properties is verified.

Common examples of such a category of additives are the thickeners. Thickeners are substances, which increase the viscosity of a liquid mixture without substantially modifying any other properties. They usually consist of synthetic as well as natural polymers, and they are used in the range of $0.2-2 \%$ in mole percentage.

In this sub-task, all the necessary product target properties $\left(\zeta_{4}\right)$ are calculated using rigorous 1 mixture property models and, if the calculated properties are found to be out of the boundaries of acceptance, the candidate formulation is rejected. It is then necessary to identify the reason for this discrepancy and find the source. Corrective actions are taken going backward in the modelbased stage. That is, apply again task 2.3. If no possible corrections can alter the product target properties enough to match the given boundaries of acceptance, then task 2.2 is applied again, and the rigorous models need to be used in the EMUD algorithm. 1

Some categories of additives may contain polar and/or ionic species. These chemicals, particularly II the designed surfactant system contains one or more ionic surfactants, can be responsible of () extreme changes in the stability of the product as an emulsion. Therefore, in this sub-task, the product stability as an emulsion is checked. Compared to the stability check performed in the EMUD algorithm, here all the ingredients are considered, while the model applied is the same.

If the product stability is not confirmed, corrective actions are taking by changing the selection of the additives, as those ingredients that are considered responsible of the product instability are rejected. If the product stability is confirmed, then a candidate emulsified formulated product has been generated by the model-based stage, and it needs to be validated in the experiment-based stage, outlined in the Experiment-based Stage 3 (presented in Supplementary Material). The 
Supplementary Material also presents the software tool used in this work as well a comparison of this methodology to other well-documented approaches for product design.

\section{The case-study of hand-wash detergent}

Two case studies involving the design of consumer products in the emulsified form have been previously developed and presented; a UV-sunscreen ${ }^{15}$ and a tank cleaning detergent ${ }^{16}$. The methodology we have illustrated has been applied in all cases, but no experimental validation has been made; only tasks 3.1 and 3.2 of stage 3 (see Supplementary Material) have been considered, as the actual experimental work has not been performed. In this paper, we consider the case study of the design of a hand-wash detergent, in the emulsified form, with emollient action. This is a complete case study, meaning that the whole methodology has been applied and that the experimental work has been planned and performed accordingly.

\section{Stage 1: Problem Definition}

Commercial hand-washes, as well as industrial detergents, are nowadays tailor-made designed in Order to satisfy a wide variety of consumer assessments. That is, the ingredients of the formulation are carefully selected in order to provide highly focused performances. Based on different surveys and other sources, the consumer needs that have been identified are listed in Table 1. The consumer need for cleaning performances is here divided in terms of three main constituents (wetting of the substrate/skin, dissolution of the dirt, suspension of the dirt) for a better identification of the relative target properties. In this table the main consumer needs $\left(\Psi_{\mathrm{m}}\right)$ as well as the secondary consumer needs $\left(\psi_{\mathrm{s}}\right)$ are listed.

Then, for Target Properties I (not modelled), eight classes of ingredients $\left(\xi_{i}\right)$ are required for the satisfaction of the consumer needs of Table 1 . They are listed in Table 2. Two surfactants are chosen because ionic surfactants are recognized to produce higher amounts of foam, as opposed 
to non-ionic surfactants, while non-ionic surfactants are usually milder on the skin. Therefore, both are selected.

Active ingredients and additives are defined by following the distinction between main $\left(\psi_{m}\right)$ and secondary $\left(\psi_{s}\right)$ consumer needs, respectively. According to such a classification (see Table 1$)$, ionic and non-ionic surfactants are defined as the active ingredients of this product, while an antibacterial agent, an aroma, a colorant, a preservative and a softener are defined as additives. Table 3 gives the relationships between consumer needs, target properties and categories of target properties. Among the thirteen target properties $\left(\zeta_{i}\right)$, seven are relative to the active ingredients $\left(\zeta_{1}\right)$, two are relative to the overall product $\left(\zeta_{4}\right)$, two are relative to all the formulation ingredients $\left(\zeta_{3}\right)$ and the remaining are related to the additives $\left(\zeta_{2}\right)$.

Besides the list of necessary classes of chemicals, also a set of target thermo-physical properties is collected from the consumer needs listed in Table 1 . Thirteen target properties (that is, target properties $I, \zeta_{i}$ ) are necessary for this case study and they are given in Table 4 , together with the boundary values.

each of the target property, a target value and/or boundary of acceptance have been set, for ingredient screening and selection reasons (see Table 4).

It needs to be noticed that the boundaries of acceptance of the Hansen solubility parameters are defined through the Hansen solubility parameters of the proteins of the skin. Moreover, two different boundaries of acceptance are set for the surface tension, as they refer to different active ingredients (i.e. different consumer needs, and hence target properties).

In summary, the output (results) of Stage 1 as described above in relation to an emulsified handwash is given in Table 5.

Some explanations of the values given in Table 5 are provided below:

This article is protected by copyright. All rights reserved. 
1. Foam-ability. The modelling of the formation of foam is fairly complex. Several works have been conducted in the recent years, developing a variety of models needing a very large number of properties to be initialized. Moreover, some of these properties are extremely hard to be calculated and even measured. For these reasons, it has been decided to apply a qualitative method for the foam-ability. Most of the above mentioned works, in fact, agreed that the lower the surface tension, the higher the foam-ability. But the role of the surfactant has also been recognized in the fact that the higher the number of micelles of the emulsion, the higher its foam-ability. In fact, the foam-ability has been found to be related to the number of stable micelles in the product, as a large number of micelles is assumed to oppose to the diffusion of air bubbles in the liquid, thus leading to a larger amount of foam produced. Therefore, arbitrary boundaries have been set on the surface tension of the overall product and on the critical micelle concentration of the surfactant (the lower the CMC, the higher the number of micelles, at the same concentration of surfactant), as a qualitative translation of the foam-ability into target properties and numerical constraints.

2. Skin irritability. The irritability of the skin is hard to be modelled and it has been decided that the $\mathrm{pH}$ and solubility properties play the main role here. The $\mathrm{pH}$ of the overall product needs to be fairly close to the $\mathrm{pH}$ of the skin, in order to be mild when spread, and it is necessary that every ingredient of the formulation (in particular the surfactants) cannot dissolve the proteins of the skin. This last constraint is translated into specific boundaries on the Hansen solubility parameters, which need to be "far" from those characterizing the proteins of the skin.

3. Cleaning performance. The cleaning performances have also been modelled with some assumptions. The complex process of the detergency has been divided into its three main phenomena: wetting, removal and suspension. The first phenomena can occur only when the surface tension of the product is below the critical surface tension of the surface to be 
cleaned, therefore a boundary on the surface tension can be set. In relation to the removal of the dirt from a surface, instead, the work of adhesion is considered the driving force. This can be calculated from the surface tension of the product and the interfacial tension between the aqueous and the organic phases of the product. This last property can be estimated itself from the surface tension values of the two phases, therefore boundaries on the surface tension can be set. Finally, the suspension is assumed to be driven only by the surfactant. That is, the prevention of the re-deposition of the dirt on the skin is assumed to be connected to the stability of the emulsion formed by the surfactant, where the dirt is expected to represent the dispersed organic phase. As a consequence, an adequate numerical constraint on the hydrophilic-lipophilic balance is set.

4. Stability. The assessment of the stability of the product as an emulsion also consists of several factors. Critical properties of the surfactants (such as the cloud point and the Krafft temperature) need to be "far" from those of standard use of the product. Moreover, the overall composition of the formulation can also influence the stability of the product, therefore the hydrophilic-lipophilic deviation needs to be well above (or below) zero. Therefore, numerical constraints on the above mentioned properties are set, and the need for a preservative in the formulation is also added to improve its stability.

5. Remaining properties. Finally, the translation of spread-ability in terms of overall density and viscosity, of safety in terms of flash point, and of toxicity in terms of lethal concentration is quite common and it has been already discussed in many previous works ${ }^{4,5,10,11,16,17}$. All the remaining consumer assessment cannot be directly translated into target properties, and they are then connected to the need for specific classes of ingredients, as seen from Tables 2 and 5. 


\section{Stage 2: Model-based Stage}

Model-based techniques are adopted in this stage for generating a candidate emulsified formulated product to be further verified in the experiment-based stage.

\section{Task 2.1}

The candidate Active Ingredients have been first screened using two databases; the ionic surfactants database, and the non-ionic surfactants database.

In order to provide foam-ability and cleaning performances, surfactants are necessary. From the knowledge base, however, it is known that ionic surfactants are usually more efficient for the production of foam, while non-ionic surfactants are usually preferred for their mildness on the skin. For these reasons, a mix of one ionic surfactant and one non-ionic surfactant is desired, where both chemicals are expected to satisfy the same numerical boundaries set for both the foam-ability and the non-irritability of the skin.

(1)

In relation to the ionic surfactants, constraints on the surface tension $(\sigma<25 \mathrm{mN} / \mathrm{m})$, on the critical micelle concentration and on the Krafft temperature are applied, while for the non-ionic (2) surfactants, constraints on the surface tension $(\sigma<55 \mathrm{mN} / \mathrm{m})$, on the Hildebrand solubility parameter, on the hydrophilic-lipophilic balance and on the cloud points are considered. For both active ingredients, in addition, the constraints on the toxicity parameter and on the flash point are also set. A short-list of candidate ionic and non-ionic surfactants is then generated via ICASProCAMD.

The effectiveness is chosen as the selection criteria for the active ingredient. In relation to the ionic surfactant, the lower the surface tension and the critical micelle concentration, the higher the performance; while for the non-ionic surfactants, the effectiveness is identified with the target values set for the Hildebrand solubility parameter. In both cases, when two or more candidate 
ingredients have comparable effectiveness, the cheapest solution is selected, also based on market availability. As a result, sodium dodecyl sulfate and tween 60 are selected as the best ionic and non-ionic surfactants, respectively.

Of course, using somewhat different ranges for the key properties, alternative choices for surfactants may be obtained (e.g., as shown in reference 16). Sodium laureth sulfate and octyl esaethylene oxide could be alternatively obtained as the two Al. The same is true also for the other components of the finally emulsified product, especially the diverse additives.

I The retrieved/predicted thermo-physical properties of the two (finally chosen) active ingredients are given in Table S1, calculated by means of pure component property models, if necessary.

Hand-wash products are usually oil-in-water emulsions, therefore it is necessary to retrieve one database for the aqueous solvent phase, and one for the organic solvent phase. As the aqueous solvent phase, water is chosen as the product is directly applied on the skin, and the nonirritability of the skin is one of the requirements. In relation to the organic solvent phase, 1

vegetable oils are often used in the cosmetic products for their moisturizing properties as well as . Imic ir pleasant aroma. The relative database is then retrieved for this case study.

()

Task 2.2

The EMUD (CAMD) algorithm is employed for the selection of the appropriate solvent mixture.

The constraints that have been set in the problem definition stage are employed, in relation to the databases selected and the property models chosen. The list of constraints on the pure and mixture properties is as follows:

\section{- $\quad$ Pure component properties}

Aqueous solvent phase: $\quad-\log \left(\mathrm{LC}_{50}\right)>3.16 \mathrm{~mol} / \mathrm{m}^{3}$ and $\mathrm{T}_{\mathrm{f}}>70^{\circ} \mathrm{C}$ 
Organic solvent phase: $\quad-\log \left(\mathrm{LC}_{50}\right)>3.16 \mathrm{~mol} / \mathrm{m}^{3}$ and $\mathrm{T}_{\mathrm{f}}>70^{\circ} \mathrm{C}$

- Mixture properties with linear mixing rule

$$
\begin{aligned}
& 30<\mathrm{V}_{\mathrm{m}}<150 \mathrm{l} / \mathrm{kmol} \\
& \delta_{\mathrm{D}}<20.4 \text { and } \delta_{\mathrm{D}}>24.4 \mathrm{MPa}^{1 / 2} \\
& \delta_{\mathrm{P}}<7.8 \text { and } \delta_{\mathrm{P}}>11.8 \mathrm{MPa}^{1 / 2} \\
& \delta_{\mathrm{H}}<9.9 \text { and } \delta_{\mathrm{H}}>13.9 \mathrm{MPa}^{1 / 2} \\
& \mathrm{C}_{\text {surf }} \geq 10 \cdot \mathrm{CMC}_{\text {surf }}
\end{aligned}
$$

- Mixture properties with non-linear mixing rule

$$
5<\mu<2500 \mathrm{cP}
$$

- Stability check

$$
H L D \neq 0
$$

For the list of constraints given above, the optimal emulsified solvent mixture generated through the EMUD algorithm is as follows:

- Water (aqueous solvent phase): $57 \%$ in weight;

- Jojoba oil (organic solvent phase): $27 \%$ in weight (see Table S2 for its properties);

- Sodium dodecyl sulfate (ionic surfactant): $8 \%$ in weight;

- Tween 60 (non-ionic surfactant): $8 \%$ in weight.

Rigorous models are used to verify that the solvent mixture properties satisfy the constraints previously set. The results are summarized below:

- Dynamic viscosity: 7.03 cP (lower boundary: 5; upper boundary: 2500 cP);

- Molar volume: 30.2 L/kmol (lower boundary: 30; upper boundary: 150 L/kmol);

- Hansen solubility parameters: dispersion: $27.4 \mathrm{MPa}^{1 / 2}$, polar: $14.5 \mathrm{MPa}^{1 / 2}$ and hydrogen-bonding $16.2 \mathrm{MPa}^{1 / 2}$

- Hydrophilic-lipophilic deviation: -3.1 (lower boundary: 0).

This article is protected by copyright. All rights reserved. 
These results satisfy the constraints set for the solvent mixture.

\section{Task 2.3}

Table 2 lists the need for six additives to be included in the emulsified formulated hand-wash, to fulfill the secondary consumer needs: an anti-bacterial agent, an aroma, a colorant, a cosurfactant, an emollient, and a preservative. However, the Food and Drug Administration (FDA) has recently expressed concerns over anti-microbial agents, as they are currently under investigation for potential carcinogen effects. For this reason, the anti-microbial agent is excluded 1 from the list of the necessary additives.

Four additives databases are then retrieved: the aroma database, the colorant database, the cosurfactant database, the emollient database, and the preservative database. Constraints on the pure component properties in terms of solubility parameters, flash point and toxicity parameters are set (Stage 1):

- Solubility parameter $14.4<\delta_{\mathrm{T}}<20.4 \mathrm{MPa}^{1 / 2}$ (organic phase) and
D $27.1<\delta_{\mathrm{T}}<33.1 \mathrm{MPa}^{1 / 2}$ (aqueous phase)
- Flash point $\mathrm{T}_{\mathrm{f}}>70^{\circ} \mathrm{C}$
- Toxicity parameter $\quad-\log \left(\mathrm{LC}_{50}\right)>3.16 \mathrm{~mol} / \mathrm{m}^{3}$

Four short-lists of candidate additives are then generated accordingly.

\section{()}

The criteria chosen for the selection of the best candidate additives is the cost. The cheapest ingredients satisfying the constraints set above is selected. The four candidate additives are then:

- Aroma: Orange sweet oil

- Colorant: Orange colorant

- Co-surfactant: Propylene glycol

- Emollient: Polyquaterium-7

This article is protected by copyright. All rights reserved. 
- Preservative: Sodium benzoate

The target properties of the selected additives are retrieved from the databases, or estimated with property models and they are given in Table S3.

The above results need verification through rigorous mixture property model based calculations for the target mixture properties. In fact, the addition of active ingredients and additives to the emulsified solvent mixture may have altered the emulsion properties, therefore it is necessary to verify that the product properties still match the constraints.

The results of the calculations are summarized below:

- Viscosity: 14.11 cP (lower boundary: 5; upper boundary: 2500 cP);

- Molar volume: 32.7 L/kmol (lower boundary: 30; upper boundary: 150 L/kmol);

The results obtained are in agreement with the constraints previously set.

\section{Task 2.4}

1

Finally, the product stability should be evaluated. The HLD value of the product is calculated, to ck if the addition of active ingredients and additives has influenced the stability of the product as an emulsion. The new calculated HLD value is -3.8 , showing a decrease compared to the solvent mixture, indicating that the stability of the product has not been compromised. The final candidate emulsified formulated product details obtained in the model-based design stage is given in Table 6. The experiment-based stage results, presented in Supplementary Material, result in a slightly different formulation, also presented in the Supplementary Material. 


\section{Conclusions and outlook for the future}

An integrated methodology for emulsified formulated product design has been proposed in this

(1)

work. It consists of three stages: a problem definition stage, a model-based design stage, and an experiment-based verification stage. In the first stage, thanks to the knowledge-base, the consumer needs are collected and translated into a set of necessary classes of ingredients and of target thermo-physical properties, with target values and/or boundaries of acceptance. The 1

second stage applies computer-aided techniques, structured databases, property models and the second stage applies computer-aided techniques, structur EMUD algorithm to generate a candidate formulation to be verified by means of experiments, from the information obtained in the previous stage. The third stage employs experimental techniques for verification of the candidate product. The properties and performances of the proposed formulation are assessed and, in case there is no match between any of the measured property or performance and its prediction, a list of actions is generated with the knowledge-base and applied for refining the proposed formulation.

(2)

developed. The EMUD algorithm is a multi-level algorithm, based on the reverse approach: given the desired set of target property values, the algorithm identifies the emulsified solvent mixtures (that is, an aqueous solvent, an organic solvent, and a surfactant system) matching the constraints. The constraints are applied at four different levels: first, constraints on pure component properties are applied, and the candidate ingredients are screened on this basis; then, constraints on mixture properties described by the linear mixing rule are employed and the compositions minimizing the cost are identified. Then, constraints on mixture properties described by non-linear mixing rules are applied, and finally, the stability of the candidate solvent mixture in the emulsified form is assessed by means of a correlation.

This article is protected by copyright. All rights reserved. 
A systematic framework for the design and analysis of emulsified formulated products has been included as a new template to the existing in-house software; the virtual Product-Process Design Laboratory, thus extending its application range. The framework is based on the problem definition and model-based design stage of the methodology for emulsified formulated product design, and it collects the knowledge-base, the databases, the models and the algorithm developed in this work.

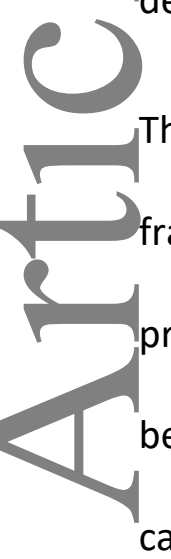

The systematic methodology for emulsified formulated product design and the corresponding framework, have been tested for a specific case study (detergent hand-wash) of a consumer product. For the first time, the whole methodology has been applied and a final prototype has also been manufactured. The results confirm that the integrated methodology developed in this work can be successfully employed for the screening of thousands of alternatives, and for the generation of a short-list of promising candidates. Some changes have been proposed in relation

(1) to some property models that should be improved for a more reliable estimation of the product Jproperties.

W/rile the proposed framework is overall satisfactory, future studies which could further improve (1) the framework should focus on the knowledge-base associated to the crucial stages of problem definition, development of quantitative models for cosmetic and sensorial properties and relating them to thermo-physical properties, need for accurate and predictive thermodynamic models for surfactant solutions. The latter could be regarded as an advance in the understanding and modeling of emulsified products, replacing some of the heuristics and correlations currently used.

Finally, when designing structured products as emulsified formulations, it is recommended that the product and the manufacturing process are designed simultaneously ${ }^{19}$. Structured products, in fact, achieve their properties through an internal structure that is determined by the interaction of its component and the manufacturing process. The recent advances achieved by the process 
systems engineering community in the area of the super-structure optimization can be applied to the solution of such a complex problem, as long as the necessary product and process models are available. The latter is often a great challenge as, for many processing steps, the needed simulation models do not exist to optimize the process or improve the known processing routes ${ }^{22}$. Still, the integration of computer-aided product design, product verification and its manufacturing process should be the ultimate target, thus including also process design and commercial feasibility evaluation. These last two steps have not been part of the scope of the current work and to our knowledge, essentially no published work has included all the above mentioned steps of complex product development. Thus, there is room for both improvements and further developments.

\section{Acknowledgment}

The authors are grateful to Dr. Sawitree Kalakul for her contributions related to the VPPD-Lab software.

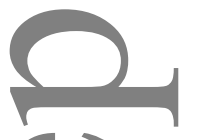

()

\section{Literature Cited}

1. Reklaitis GV. Perspectives on Systems Engineering advances in Process and Product Design. Proceedings of the $8^{\text {th }}$ International Conference on Foundations of Computer-Aided Process Design. Book Series: Computer Aided Chemical Engineering. 2014; 34: 1-4.

2. Karunanithi, AT, Achenie, LEK, Gani R. Optimal (solvent) design through a decomposition based CAMD methodology. Conference: 14th European Symposium on Computer Aided Process 
Engineering (ESCAPE-14) Location: Lisbon, PORTUGAL Date: MAY 16-19, 2004. Book series: Computer-Aided Chemical Engineering. 2004; 18: 217-222.

3. Villadsen J. Putting structure into chemical engineering - Proceedings of an industry/university conference. Chemical Engineering Science. 1997; 52: 2857-2864.

4. Gani R. Chemical product design: challenges and opportunities. Computers and Chemical Engineering. 2004; 28: 2441-2457.

5. Cussler EL., Moggridge GD. Chemical product design. Cambridge University Press, Cambridge, ME, 2011.

6. Wesselingh, JA., Kiil, S., Vigild, ME. Design \& Development of Biological, Chemical, Food and Pharmaceutical Products. Wiley, 2007.

7. Smith BV, lerapetritou M. Sensitivity-Based Product Portfolio and Design Integration.
8nd.Eng.Chem.Res. 2011; 50: 3919-3927.
8. Bernardo FP, Saraiva PM. A Conceptual Model for Chemical Product Design. AIChE J. 2015; 61: Don'-815.

(1)

9. Bagajewicz M., Hill S, Robben A, Lopez H, Sanders M, Sposato E, Baade C, Manora, S, Coradin, JH. Product Design in Price-Competitive Markets: A Case Study of a Skin Moisturizing Lotion. AIChE J. 2011; 57: 160-177.

10. Gani R, Ng KM. Product Design - From Molecules to Formulations to Devices. Conference: 8th International Conference on the Foundations of Computer-Aided Process Design (FOCAPD) Location: Cle Elum, WA Date: JUL 13-17, 2014. Book Series: Computer Aided Chemical Engineering. 2014; 34: 108-123.

This article is protected by copyright. All rights reserved. 
11. Ng KM, Gani R, Dam-Johansen K. Chemical product design. Toward a perspective through case studies. Elsevier, Amsterdam, the Netherlands, 2007.

12. Conte E, Gani R, Ng KM. Design of formulated products: a systematic methodology. AlChE Journal. 2011; 57: 2431-2449.

13. Mattei M., Kontogeorgis GM, Gani R. A comprehensive framework for surfactant selection and design for emulsion based chemical product design. Fluid Phase Equilibria. 2014; 362: 288-299.

14. Mattei M, Yunus NA, Kalakul S, Kontogeorgis GM, Woodley JM, Gernaey KV, Gani R. The Virtual Product-Process Design Laboratory for Structured Chemical Product Design and Analysis. 24TH EUROPEAN SYMPOSIUM ON COMPUTER AIDED PROCESS ENGINEERING, PTS A AND B. Book Series: Computer Aided Chemical Engineering. 2014; 33: 61-66.

15. Mattei M, Kontogeorgis GM, Gani R. A Systematic Methodology for Design of Emulsion Based Chemical Products. Conference: 11th International Symposium on Process Systems Engineering (PSE) Location: Singapore, SINGAPORE Date: JUL 15-19, 2012. Book Series: Computer Aided Chemical Engineering. 2012; 31: 220-224.

16. Mattei M, Hill M, Kontogeorgis GM, Gani R. Design of an Emulsion-based Personal Detergent through a Model-based Chemical Product Design Methodology. Conference: 23rd European Symposium on Computer Aided Process Engineering (ESCAPE) Location: Lappeenranta Univ Technol, Lappeenranta, FINLAND Date: JUN 09-12, 2013. Book Series. Computer Aided Chemical Engineering. 2011; 32: 817-822.

17. Mattei M, Conte E, Kontogeorgis GM, Gani R. Prediction of thermophysical properties of liquid formulated products. Book Chapter in Brockel U., Meier W. And Wagner G.; Product Design and Engineering: Formulation of Gels and Pastes. Wiley-VCH, 2013. 
18. Mattei M, Kontogeorgis GM, Gani R. Modeling of the Critical Micelle Concentration (CMC) of Nonionic Surfactants with an Extended Group-Contribution Method. Industrial and Engineering Chemistry Research. 2013; 52: 12236-12246.

19. Zhang L, Babi, DK, Gani, R. New vistas in chemical product-process design. Annual Review of Chemical and Biomolecular Engineering. 2016; 7: 557-582.

20. Zhang L, Fung KY, Wibowo C, Gani, R. Advances in Chemical Product Design. Reviews in Chemical Engineering. 2018; 34: 319-340.

21. Tula, AK, Eden, MR, Gani, R. Process synthesis, design and analysis using a process-group contribution method. Computers \& Chemical Engineering, 2015; 81: 245-259.

22. Hill, M. Product and process design for structured products. AIChE J. 2004; 50: 1656-1661. 


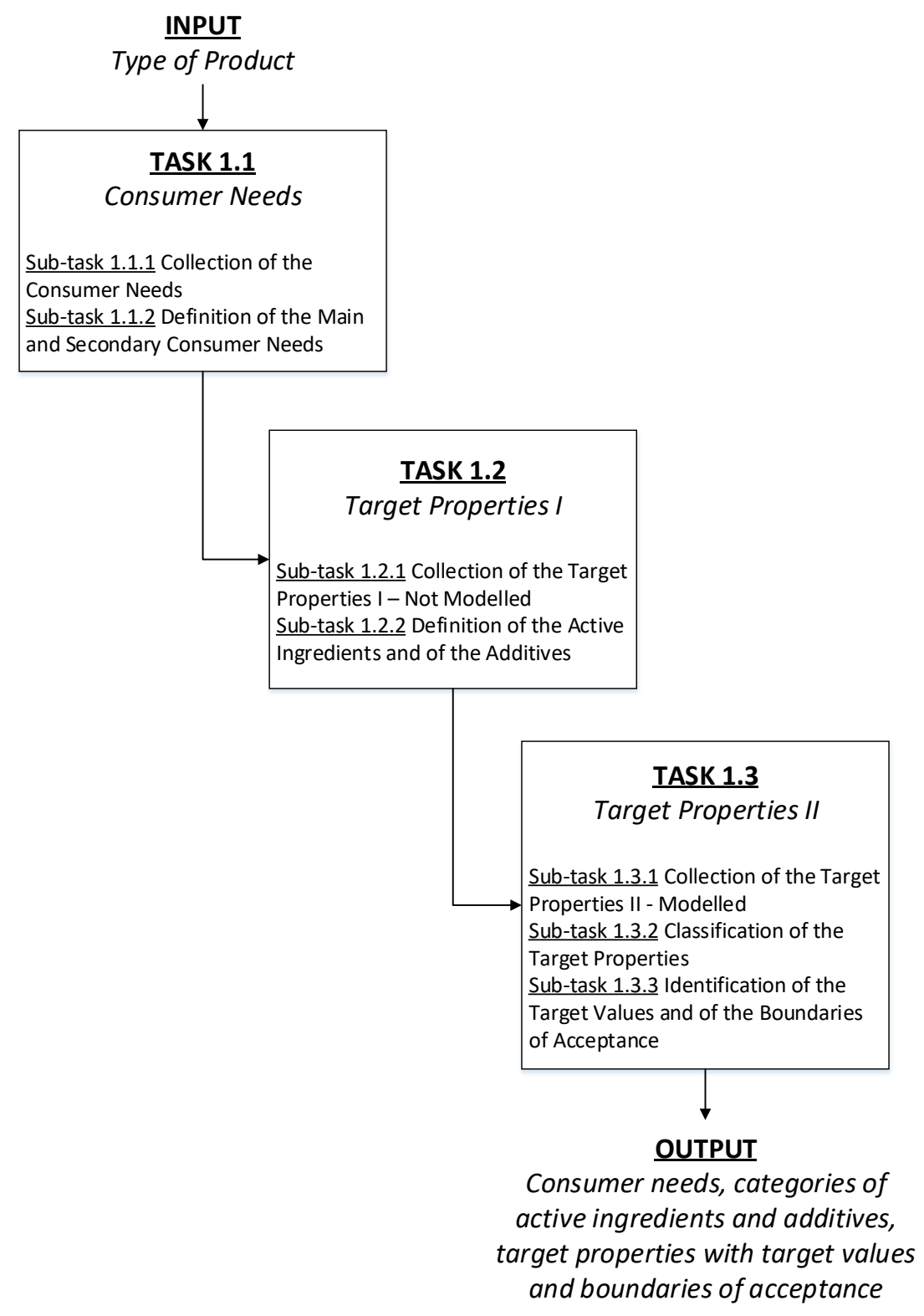

Figure 3. The work-flow of the problem definition stage for the design of emulsified formulated products.

This article is protected by copyright. All rights reserved. 
INPUT

Categories of active ingredients and additives, target properties with target values and boundaries of acceptance
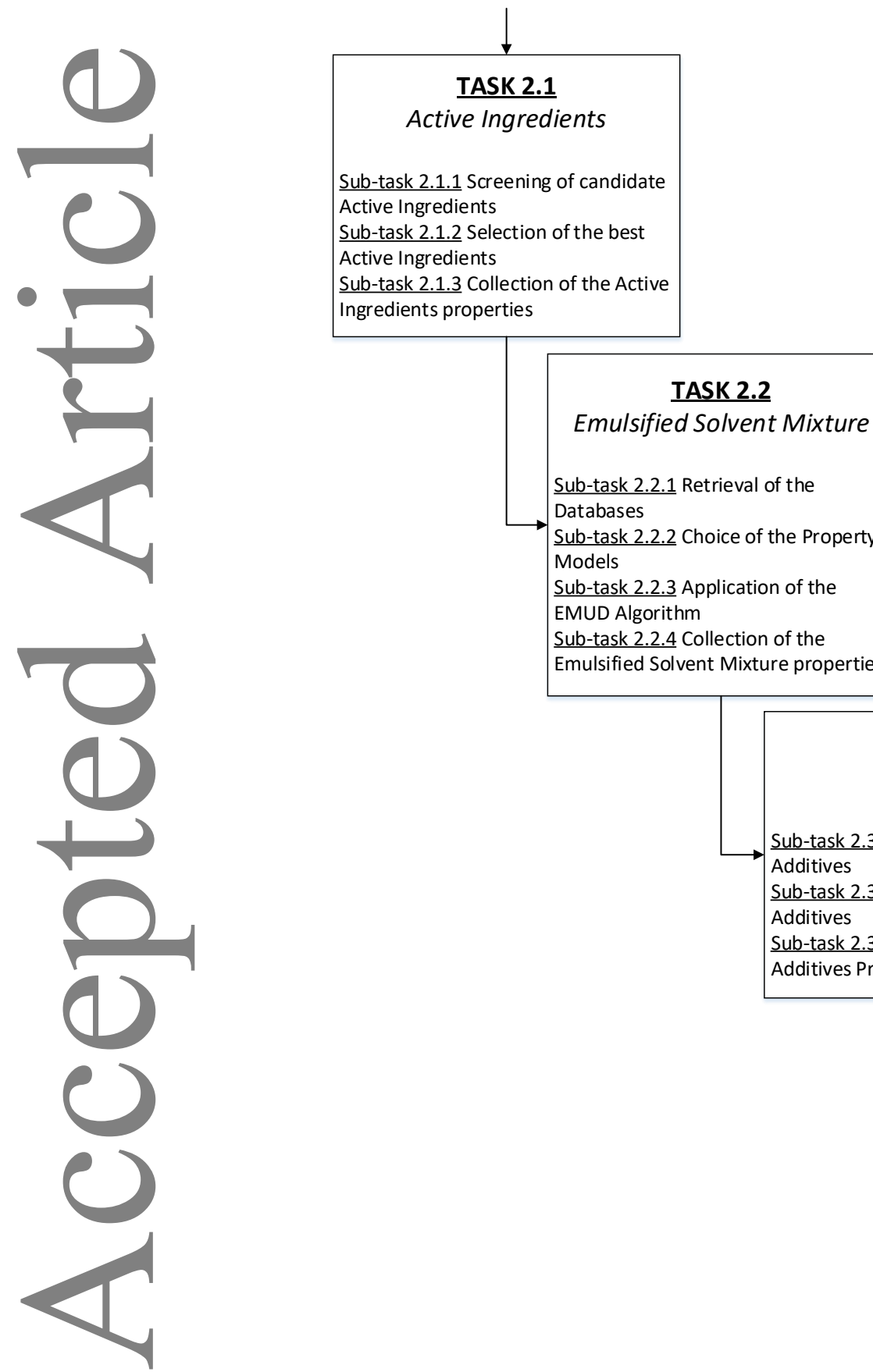

Sub-task 2.2.1 Retrieval of the Databases

Sub-task 2.2.2 Choice of the Property Models

Sub-task 2.2.3 Application of the EMUD Algorithm

Sub-task 2.2.4 Collection of the

Emulsified Solvent Mixture properties

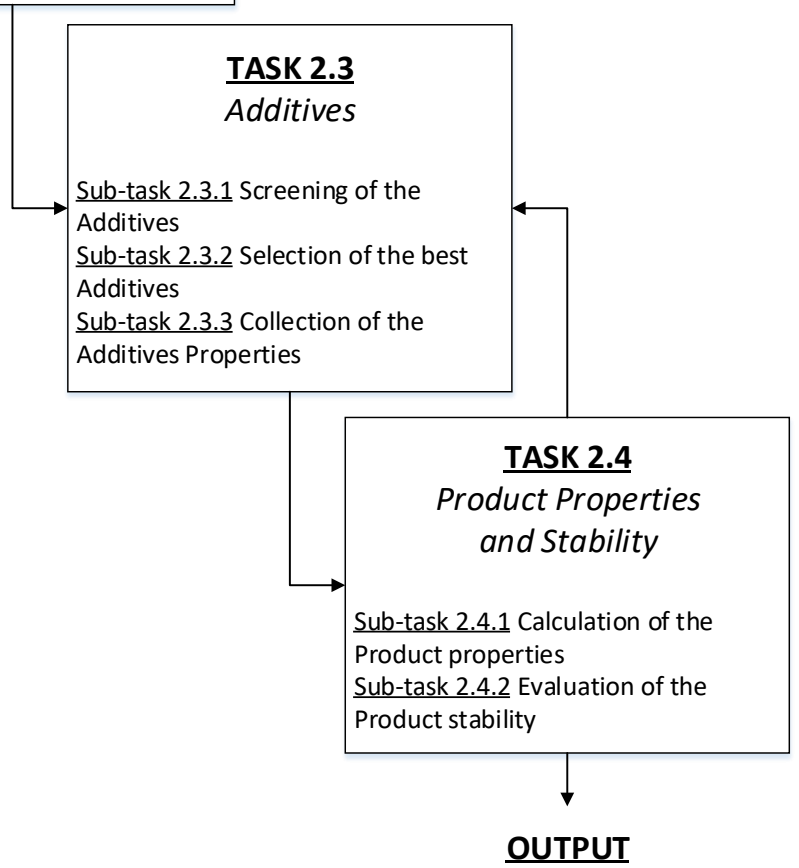

Candidate Product with thermo-physical properties

Figure 4. The work-flow of the model-based stage 2 for the design of emulsified formulated products. 
Table 1. Distinction between Main and Secondary Consumer Needs for a Hand-Wash in the Emulsified Form

Consumer Needs $\left(\psi_{\mathrm{i}}\right)$
High foam-ability
Non irritability of the skin
Wetting of the substrate
Dissolution of the dirt $\left(\Psi_{\mathrm{M}}\right)$
Suspension of the dirt $\left(\psi_{\mathrm{s}}\right)$
Spread-ability
Good stability
Anti-bacterial performances
Pleasant color
Pleasant odor

This article is protected by copyright. All rights reserved. 
Table 2. Collection of the Classes of Ingredients and Distinction between Active Ingredients and Additives for a Hand-Wash in the Emulsified Form

\begin{tabular}{|c|c|c|}
\hline Consumer Needs $\left(\Psi_{\mathrm{i}}\right)$ & $\begin{array}{c}\text { Class of } \\
\text { Ingredients }\left(\xi_{i}\right)\end{array}$ & $\begin{array}{c}\text { Active Ingredients }\left(\xi_{A I}\right) \\
\text { or Additives }\left(\xi_{A D D}\right)\end{array}$ \\
\hline High foam-ability & Ionic Surfactant & $\xi_{\mathrm{Al}}$ \\
\hline \multicolumn{3}{|l|}{ Non irritability of the skin } \\
\hline Wetting of the substrate & Non-Ionic & \\
\hline Dissolution of the dirt & Surfactant & $\xi_{\mathrm{Al}}$ \\
\hline \multicolumn{3}{|l|}{ Suspension of the dirt } \\
\hline Spread-ability & - & - \\
\hline \multirow{2}{*}{ Good stability } & Co-surfactant & $\xi_{A D D}$ \\
\hline & Preservative & $\xi_{\mathrm{ADD}}$ \\
\hline Anti-bacterial & Anti-bacterial & \\
\hline performances & Agent & $\xi_{\mathrm{ADD}}$ \\
\hline Pleasant color & Colorant & $\xi_{A D D}$ \\
\hline Pleasant odor & Aroma & $\xi_{A D D}$ \\
\hline Pleasant skin feeling & Emollient & $\xi_{A D D}$ \\
\hline Low toxicity & - & - \\
\hline High safety & - & - \\
\hline
\end{tabular}

This article is protected by copyright. All rights reserved. 
Table 3. Collection and Classification of the Target Properties, for a Hand-Wash in the Emulsified Form

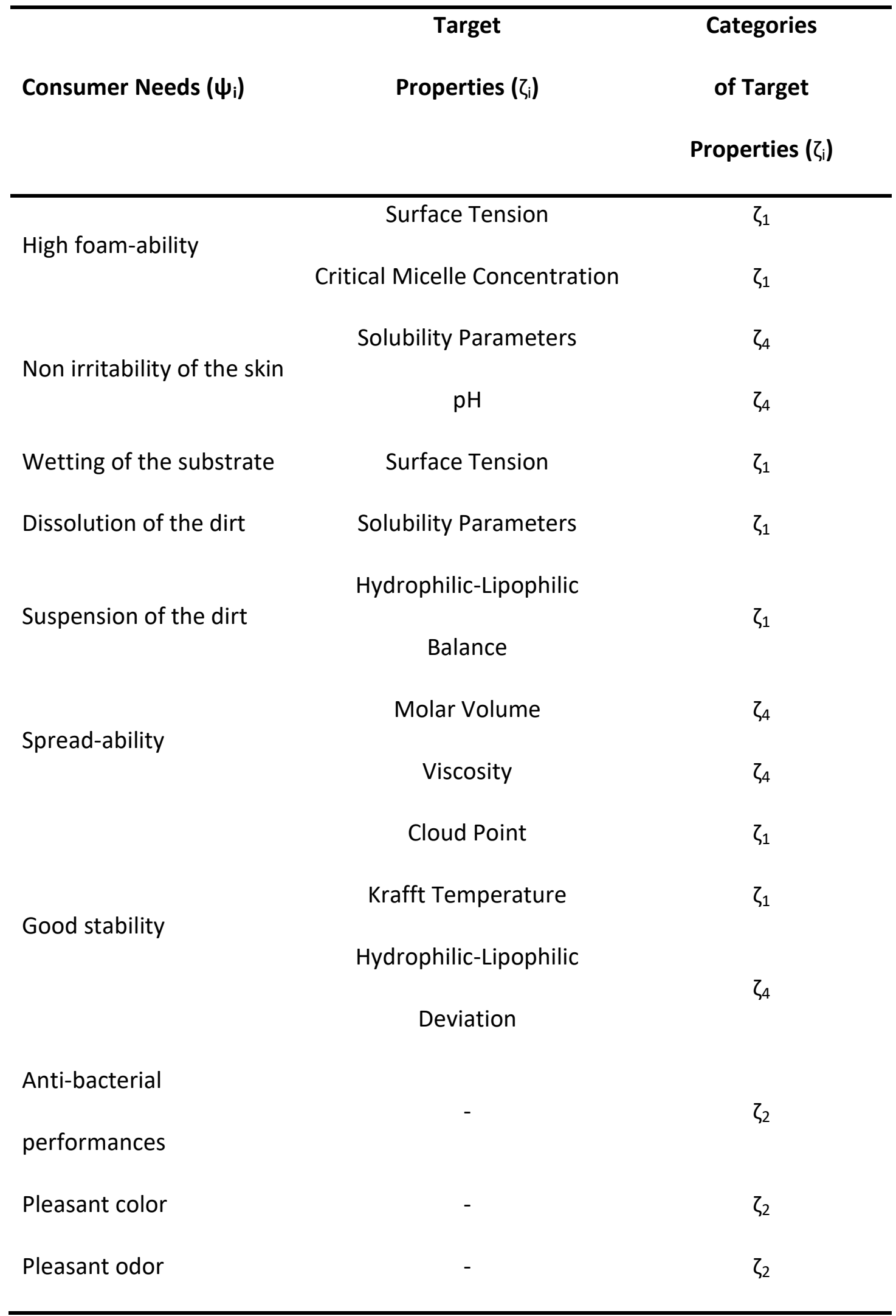

This article is protected by copyright. All rights reserved. 


\begin{tabular}{lcc}
\hline Pleasant skin feeling & - & $\zeta_{2}$ \\
Low toxicity & Toxicity Parameter & $\zeta_{3}$ \\
High safety & Flash point & $\zeta_{3}$ \\
\hline
\end{tabular}

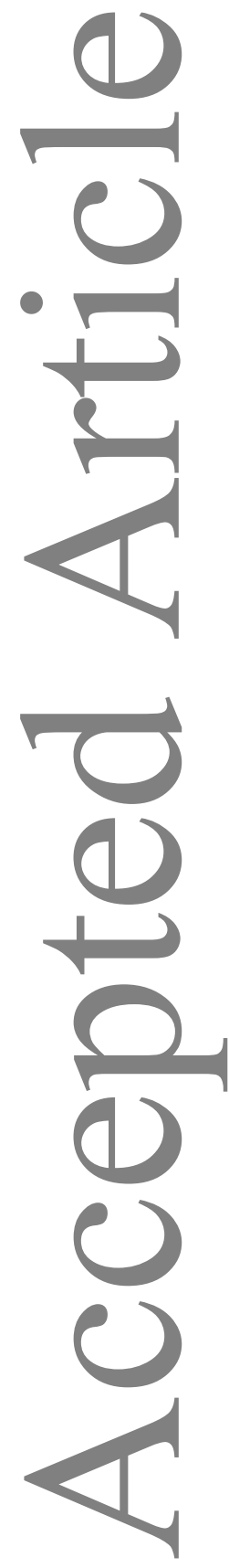

This article is protected by copyright. All rights reserved. 
Table 4. Collection of the Target Values and of the Boundaries of Acceptance of the Target Properties, for a Hand-Wash Detergent in the Emulsified Form

\begin{tabular}{|c|c|c|c|}
\hline Target Properties & Target Value & Boundaries of & Unit of \\
\hline$\left(\zeta_{i}\right)$ & $\left(\zeta_{i, T}\right)$ & Acceptance $\zeta_{\mathrm{i}, \mathrm{L}} \zeta_{\mathrm{i}, \mathrm{U}}$ & Measure \\
\hline Surface Tension & - & $\sigma<25$ & $\mathrm{mN} / \mathrm{m}$ \\
\hline \multirow[t]{3}{*}{ Critical Micelle Concentration } & - & $\mathrm{CMC}<0.01$ & $\mathrm{~mol} / \mathrm{L}$ \\
\hline & - & $\delta_{D}<20.4$ and $\delta_{D}>$ & $\mathrm{MPa}^{1 / 2}$ \\
\hline & - & 24.4 & $\mathrm{MPa}^{1 / 2}$ \\
\hline \multicolumn{2}{|l|}{ Solubility Parameters } & $\delta_{p}<7.8$ and $\delta_{p}>11.8$ & $\mathrm{MPa}^{1 / 2}$ \\
\hline & & $\delta_{H}<9.9$ and $\delta_{H}>13.9$ & {$[-]$} \\
\hline & $\mathrm{pH}=5.5$ & $4.5<\mathrm{pH}<8$ & \\
\hline Surface tension & - & $\sigma<55$ & $\mathrm{mN} / \mathrm{m}$ \\
\hline Solubility Parameters & $\delta_{\mathrm{T}}=17.7$ & $14.7<\delta_{\mathrm{T}}<20.7$ & $\mathrm{MPa}^{1 / 2}$ \\
\hline Hydrophilic-Lipophilic & - & HLB $>12$ & {$[-]$} \\
\hline \multicolumn{4}{|l|}{ Balance } \\
\hline Molar Volume & - & $30<V_{\mathrm{m}}<150$ & $\mathrm{~L} / \mathrm{kmol}$ \\
\hline Viscosity & - & $5<\mu<2500$ & $\mathrm{cP}$ \\
\hline Cloud Point & - & $C P>70$ & ${ }^{\circ} \mathrm{C}$ \\
\hline Krafft temperature & - & $\mathrm{T}_{\mathrm{K}}<20$ & ${ }^{\circ} \mathrm{C}$ \\
\hline Hydrophilic-Lipophilic & - & HLD $\neq 0$ & {$[-]$} \\
\hline \multicolumn{4}{|l|}{ Deviation } \\
\hline Toxicity Parameter & - & $-\log \left(L C_{50}\right)>3.16$ & $\mathrm{~mol} / \mathrm{m}^{3}$ \\
\hline Flash Point & - & $T_{f}>70$ & ${ }^{\circ} \mathrm{C}$ \\
\hline
\end{tabular}

This article is protected by copyright. All rights reserved. 
Table 5. Summary of the Results of Stage 1 for the Hand-Wash Cream

\begin{tabular}{|c|c|c|c|}
\hline & $\begin{array}{l}\text { Consumer } \\
\text { assessments }\end{array}$ & Properties considered & Constraints \\
\hline \multirow{9}{*}{$\begin{array}{l}\tilde{0} \\
\text { 岕 } \\
\frac{2}{z} \\
\vdots \\
\vdots \\
\Sigma\end{array}$} & & Surface tension & $\sigma<22 \mathrm{mN} / \mathrm{m}$ \\
\hline & Foam-ability & $\begin{array}{l}\text { Critical micelle } \\
\text { concentration }\end{array}$ & $\mathrm{CMC}<0.01 \mathrm{~mol} / \mathrm{L}$ \\
\hline & \multirow{4}{*}{ Non-irritability of } & \multirow{3}{*}{$\begin{array}{l}\text { Hansen solubility } \\
\text { parameters }\end{array}$} & $\begin{array}{l}\delta_{D}<20.4 \&> \\
24.4 \mathrm{MPa}^{0.5}\end{array}$ \\
\hline & & & $\begin{array}{l}\delta_{\mathrm{P}}<7.8 \&>11.8 \\
\mathrm{MPa}^{0.5}\end{array}$ \\
\hline & & & $\begin{array}{l}\delta_{\mathrm{H}}<9.9 \&>13.9 \\
\mathrm{MPa}^{0.5}\end{array}$ \\
\hline & & $\mathrm{pH}$ & $4.5<\mathrm{pH}<8$ \\
\hline & \multirow{3}{*}{$\begin{array}{l}\text { Cleaning } \\
\text { performances }\end{array}$} & Surface tension & $\sigma<55 \mathrm{mN} / \mathrm{m}$ \\
\hline & & Solubility parameter & $\begin{array}{l}14.7<\delta_{\mathrm{T}}<20.7 \\
\mathrm{MPa}^{0.5}\end{array}$ \\
\hline & & $\begin{array}{l}\text { Hydrophilic-lipophilic } \\
\text { balance }\end{array}$ & HLB $>10$ \\
\hline \multirow{3}{*}{ 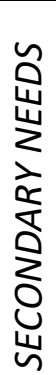 } & \multirow[t]{2}{*}{ Spreadability } & Molar Volume & $\begin{array}{l}30<\mathrm{V}_{\mathrm{m}}<150 \\
\mathrm{~L} / \mathrm{kmol}\end{array}$ \\
\hline & & Viscosity & $5<\mu<2500 \mathrm{cP}$ \\
\hline & Emulsion stability & Cloud point & $\mathrm{CP}>70^{\circ} \mathrm{C}$ \\
\hline
\end{tabular}

This article is protected by copyright. All rights reserved. 


\begin{tabular}{|c|c|c|}
\hline & Krafft temperature & $\mathrm{T}_{\mathrm{K}}<20^{\circ} \mathrm{C}$ \\
\hline & $\begin{array}{l}\text { Hydrophilic-lipophilic } \\
\text { deviation }\end{array}$ & HLD $\neq 0$ \\
\hline & $\begin{array}{l}\text { Presence of a } \\
\text { preservative }\end{array}$ & - \\
\hline $\begin{array}{l}\text { Anti-bacterial } \\
\text { performances }\end{array}$ & $\begin{array}{l}\text { Presence of an anti- } \\
\text { bacterial agent }\end{array}$ & - \\
\hline Pleasant colour & Presence of a pigment & - \\
\hline Pleasant odour & Presence of an aroma & - \\
\hline Pleasant skin feeling & Presence of a softener & - \\
\hline Safety & Flash point & $\mathrm{T}_{\mathrm{f}}>70^{\circ} \mathrm{C}$ \\
\hline Non-toxicity & Toxicity parameter & $\begin{array}{l}-\log \left(\mathrm{LC}_{50}\right)>3.16 \\
\mathrm{~mol} / \mathrm{m}^{3}\end{array}$ \\
\hline
\end{tabular}

This article is protected by copyright. All rights reserved. 
Table 6. Candidate Formulation for a Hand-Wash in the Emulsified Form

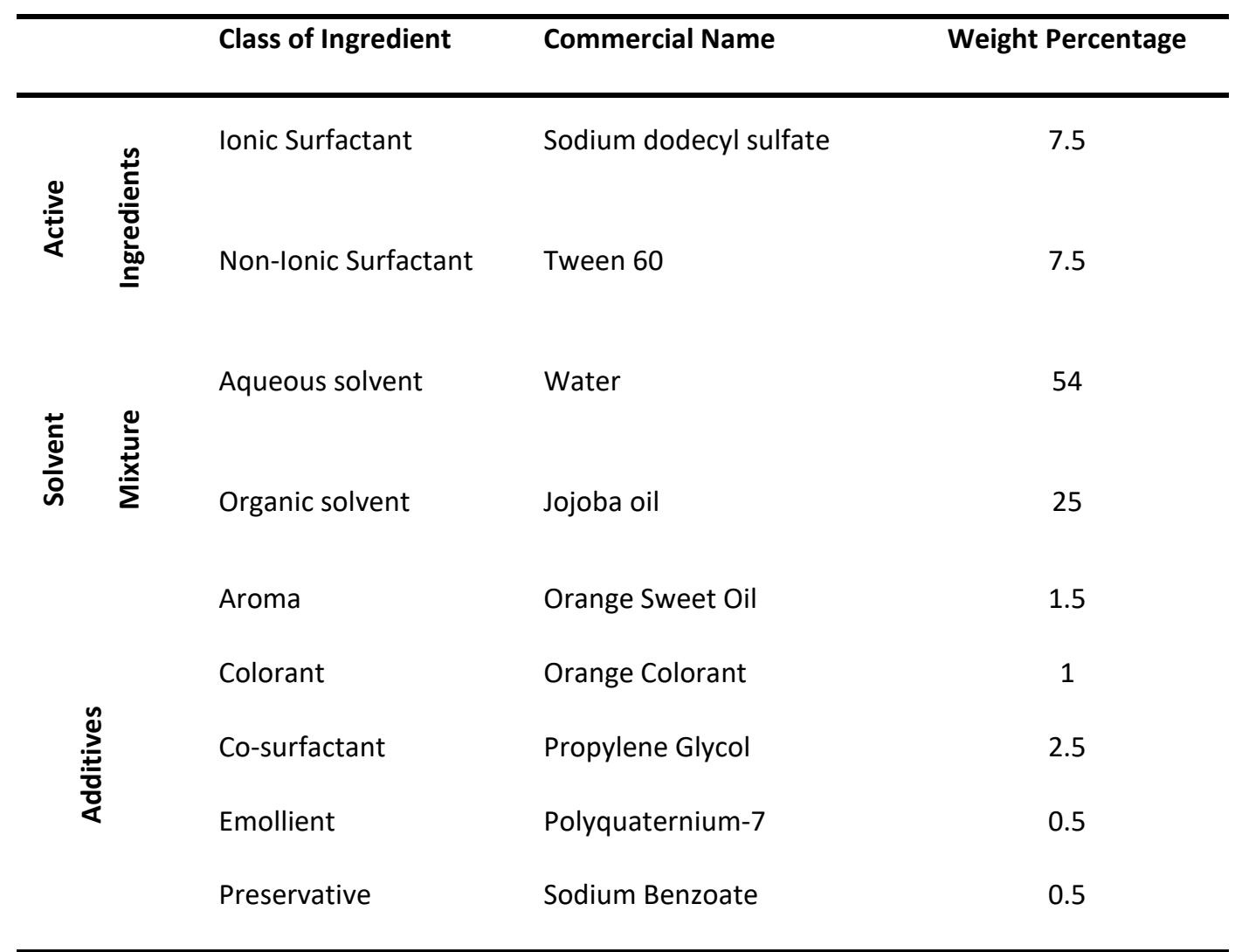

This article is protected by copyright. All rights reserved. 